\title{
Fluctuations in Pigment Epithelial Detachment and Retinal Fluid Using a Bimonthly Treatment Regimen with Aflibercept for Neovascular Age-Related Macular Degeneration
}

\author{
Martin Sebastian Zinkernagel Sebastian Wolf Andreas Ebneter \\ Department of Ophthalmology, University Hospital Bern, Bern, Switzerland
}

\section{Key Words}

Retina · Anti-VEGF · Intravitreal injections · Pigment epithelial detachment $\cdot$ Spectral domain optical coherence tomography · Atrophy

\begin{abstract}
Purpose: To assess the effect of a bimonthly treatment regimen with intravitreal aflibercept on retinal fluid and pigment epithelial detachment (PED) in patients with neovascular age-related macular degeneration (AMD). Methods: Twenty-six treatment-naive eyes of 26 patients with choroidal neovascularisation secondary to AMD were included. The patients received three initial monthly (mean 30 days) intravitreal injections of aflibercept followed by a bimonthly (mean 62 days) fixed regimen for a total of 1 year. Best-corrected visual acuity (BCVA) and optical coherence tomography (OCT) measurements were recorded at monthly intervals. In addition, the presence of intraretinal fluid (IRF) or subretinal fluid (SRF) or a combination of both as well as serous and fibrovascular PEDs were assessed. Results: The mean patient age was 80 years (range 54-93). There were 14 male and 12 female patients. The mean gain in BCVA at 1 year was 9.3 letters $(S E M \pm 3$ ) with a mean reduction of the central retinal thickness of $154 \mu \mathrm{m}$ (SEM \pm 50 ). After 3 monthly injections of aflibercept, there was resolution of IRF and
\end{abstract}

SRF in $80 \%$ of the treated eyes; the amount of fluid increased at months 4,6 and 8 with troughs in between. Whereas fibrovascular PEDs remained stable after the loading phase, serous PEDs displayed a seesaw pattern. Patients without retinal pigment epithelium (RPE) atrophy at the end of the 1-year period had significantly better BCVA compared to patients with RPE atrophy $(p=0.03)$. Conclusion: Despite significant overall BCVA gain, bimonthly intervals seem insufficient to maintain the morphological improvements after the initial loading dose with intravitreal aflibercept.

(c) 2015 S. Karger AG, Basel

\section{Introduction}

Age-related macular degeneration (AMD) is a chronic and degenerative disease and one of the leading causes of legal blindness in patients over the age of 60 years in the industrialized world [1]. The most severe vision loss occurs in the neovascular form of AMD, associated with intraretinal fluid (IRF) or subretinal fluid (SRF) caused by choroidal neovascularisation (CNV). Current treatment

Part of this work was presented at the Jules Gonin Meeting, September 4-6, 2014, Zurich, Switzerland.

\section{KARGER 125}

(c) 2015 S. Karger AG, Basel

$0030-3755 / 15 / 0000-0000 \$ 39.50 / 0$

E-Mail karger@karger.com

www.karger.com/oph
Martin S. Zinkernagel

Department of Ophthalmology, Inselspital

University Hospital Bern

$\mathrm{CH}-3010$ Bern (Switzerland)

E-Mail m.zinkernagel@gmail.com 
options for the neovascular component consist of antiangiogenic substances (anti-VEGF) that are able to reduce intraretinal and subretinal leakage and oedema. AntiVEGF therapies, such as intravitreal ranibizumab (Lucentis; Genentech, Inc., South San Francisco, Calif., USA) and aflibercept (Regeneron Pharmaceuticals, Inc., Tarrytown, N.Y., USA, and Bayer HealthCare, Berlin, Germany), have been shown to restore macular morphology and consecutively to preserve or even improve visual acuity in multiple clinical trials $[2,3]$.

To reduce the burden of monthly intravitreal injections, new regimens that may provide equivalent efficacy and anatomic control compared to monthly ranibizumab have been studied (PIER/EXCITE) [4, 5]. However, none of these regimens achieved a similar long-term vision gain compared to monthly intravitreal ranibizumab injections. More recently, the Comparison of AMD Treatments Trials (CATT) $[6,7]$ has compared monthly ranibizumab to pro re nata (PRN) regimens with monthly visits. Anatomical criteria were used to decide on the need for retreatment. Here, PRN ranibizumab was noninferior to the monthly gain, however, with a mean bestcorrected visual acuity (BCVA) gain of 1.7 letters less than that of the monthly standard treatment. With the introduction of aflibercept following the two pivotal VIEW studies [2, 3], showing non-inferiority of 8-week injections compared to monthly ranibizumab injections, the need for monthly follow-up visits became redundant. Although the VIEW studies used an 8-week (average of 56 days) retreatment regimen, a bimonthly retreatment regimen (average of 61 days) is often more practicable due to organisational reasons in real life. Aflibercept is a fusion protein of key domains from human VEGF receptors 1 and 2 [8] and has been shown to have a significantly higher binding affinity to VEGF than ranibizumab [9-11].

Here, we report on morphological and functional outcomes using a fixed bimonthly aflibercept treatment regimen at a tertiary referral centre.

\section{Methods}

\section{Participants}

In this retrospective interventional study, a consecutive series of treatment-naive patients receiving intravitreal aflibercept for exudative AMD were identified from the procedure logs at the Department of Ophthalmology of the University Hospital Bern. All patients had clinically confirmed exudative AMD and had spectral domain optical coherence tomography (SD-OCT) imaging performed before receiving their initial treatment. All patients re-

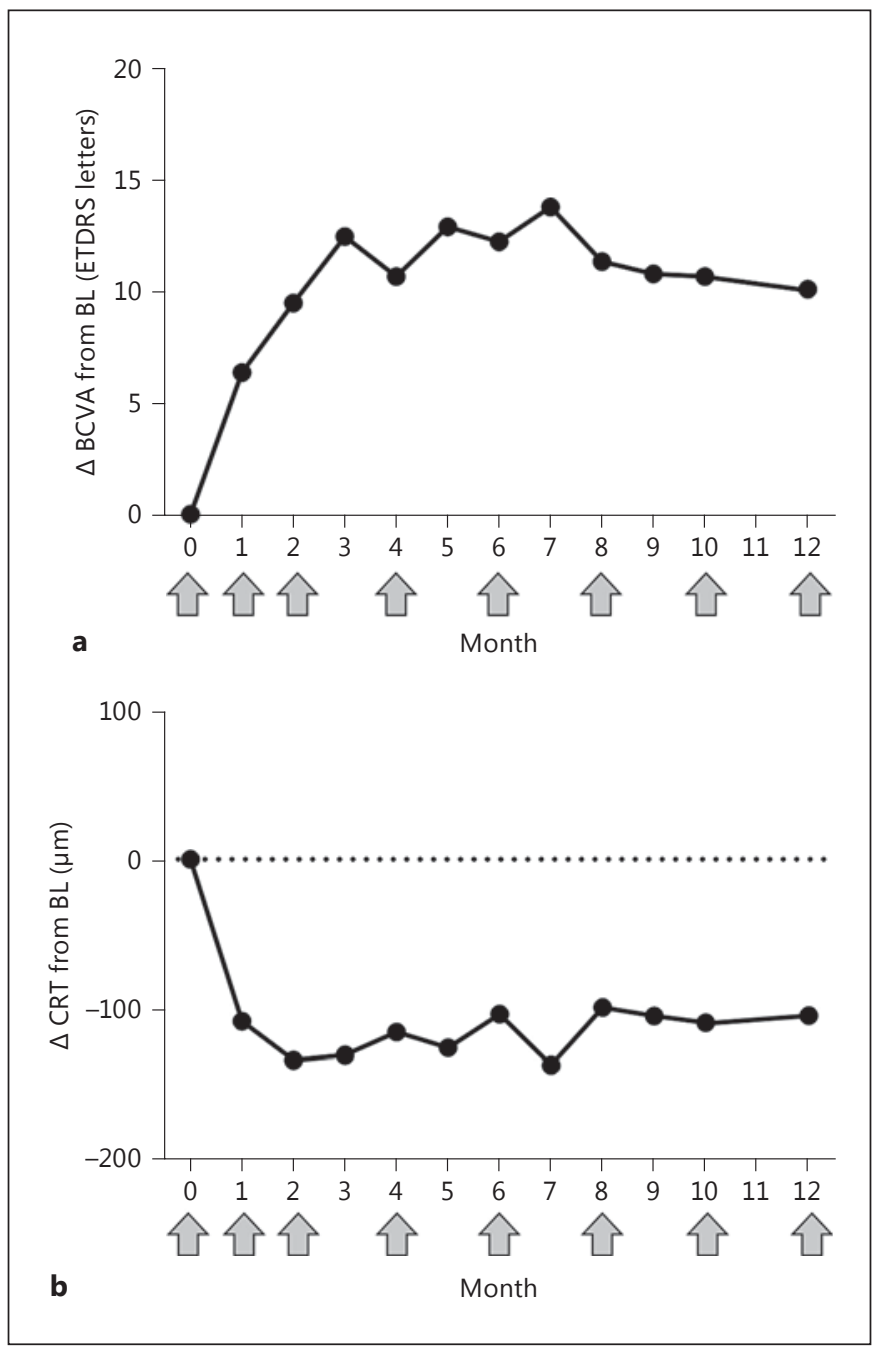

Fig. 1. Functional and anatomical results using a fixed bimonthly maintenance treatment with aflibercept for exudative AMD. a Mean change in BCVA from baseline (BL). b Mean change in CRT from baseline (BL) (arrows show injection frequency).

ceived an identical loading dose of three consecutive monthly intravitreal injections followed by bimonthly maintenance treatment with aflibercept. Ethics approval (KEK-No. 093/13) to conduct this study was obtained from the local ethics committee, which is working in accordance with ICH-GCP guidelines. The need for written consent from each individual patient was waived because of the retrospective nature of the study.

\section{Image Acquisition}

Spectralis SD-OCT (Heidelberg Engineering, Heidelberg, Germany; Software version 5.3) scans were acquired using an established protocol consisting of both cross-hair scans and volume scans. The cross-hair scan consisted of a vertical and a horizontal scan. For the volume scan of $20^{\circ} \times 20^{\circ}, 49$ frames spaced $121 \mu \mathrm{m}$ and each consisting of $512 \mathrm{~A}$-scans were acquired. The automated real-time was set between 6 and 12 scans to reduce noise. 


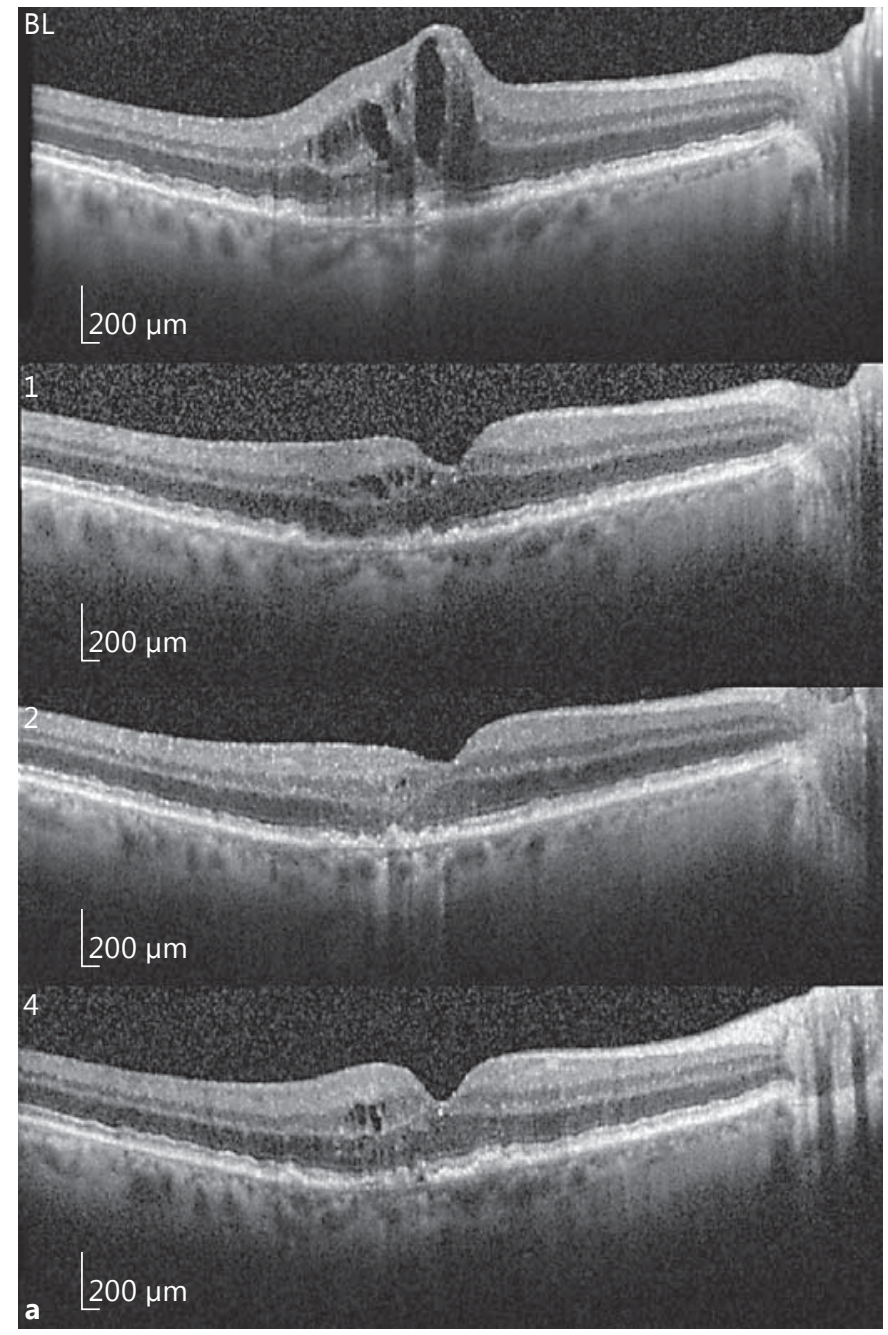

Image Analysis

Each imaging data set was reviewed by two independent assessors, and the presence of SRF or IRF was evaluated at each visit. In addition, the presence of serous (evidenced by hyporeflective internal reflectivity) or fibrovascular (evidenced by heterogeneous internal reflectivity) [12] pigment epithelial detachments (PEDs) was recorded for each monthly follow-up visit (except for month 10). Central retinal thickness (CRT) was measured automatically as the distance from the internal limiting membrane of the retina to the outer border of the retinal pigment epithelium (RPE) excluding any sub-RPE structures like CNV membranes. Using the Heidelberg Eye explorer software (version 1.7.1.0), the area (measured in square millimetre) of the PED was measured in the horizontal cross-hair OCT scan and the corresponding follow-ups. Then, the subfoveal choroidal thickness was measured from the Bruch membrane to the inner scleral margin.

In addition to monthly SD-OCT imaging, BCVA was measured monthly according to a standardized refraction procedure using ETDRS charts at 1 and $4 \mathrm{~m}$. Atrophy of the RPE within the

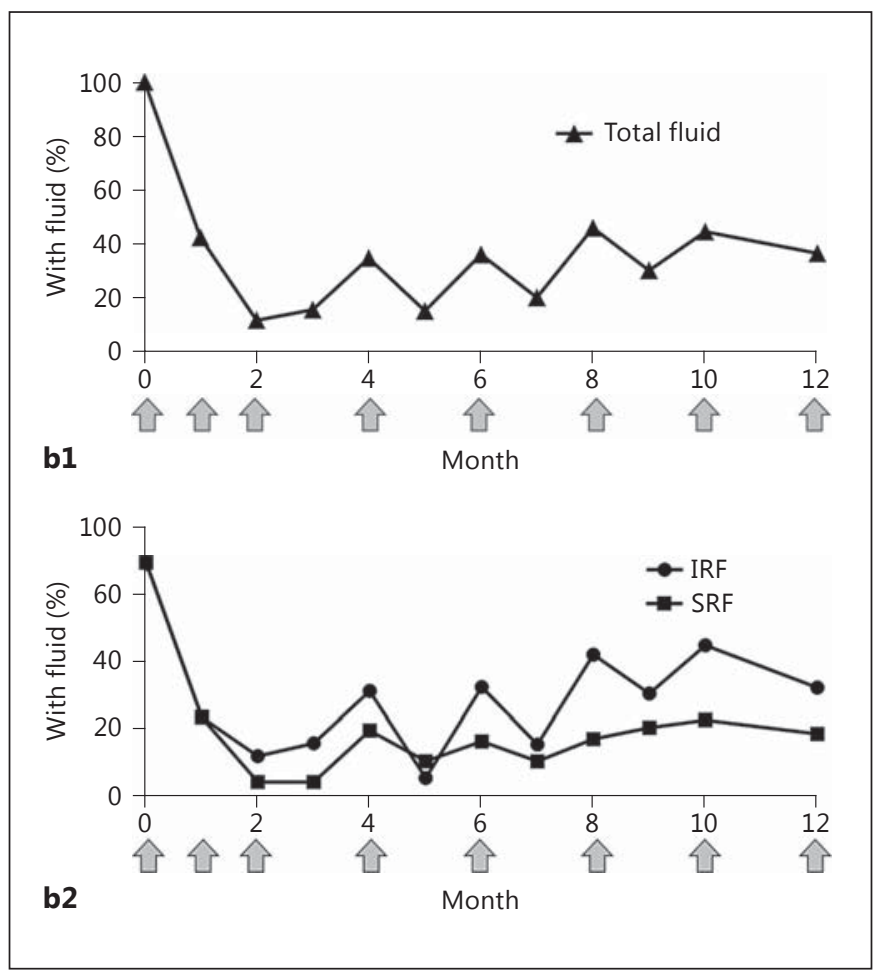

Fig. 2. The response of IRF and SRF to aflibercept using a fixed treatment regimen is presented. a Representative OCT scans at baseline (BL), months 1, 2 and 4. b1 Percentage of patients with IRF and SRF (total fluid). b2 Percentage of patients with either SRF or IRF at each month (arrows show injection frequency).

macula (defined as within the volume scan of $6 \times 6 \mathrm{~mm}$ ) was graded by two independent assessors at baseline and at year 1 using either autofluorescence or infrared OCT images. For patients developing atrophy over the follow-up period, we assessed the OCT scans performed at control visits to evaluate the time point of atrophy development.

The last observation carried forward approach was used. Twenty-four patients completed the 12 months, whereas 2 patients discontinued treatment at 10 and 9 months, respectively.

\section{Statistical Analysis}

A statistical analysis was performed using the Prism Graph $\mathrm{Pad}^{\circledR}$ commercial software package (Prism 5; GraphPad Software Inc., La Jolla, Calif., USA). BCVA and CRT data was analysed by ANOVA or paired t test as indicated (Wilcoxon matched-pairs signed-rank test for nonparametric data). For comparison between groups, an unpaired t test was employed. For correlation, Spearman nonparametric analysis $(\rho)$ was used. $\mathrm{p}<0.05$ was considered statistically significant. 


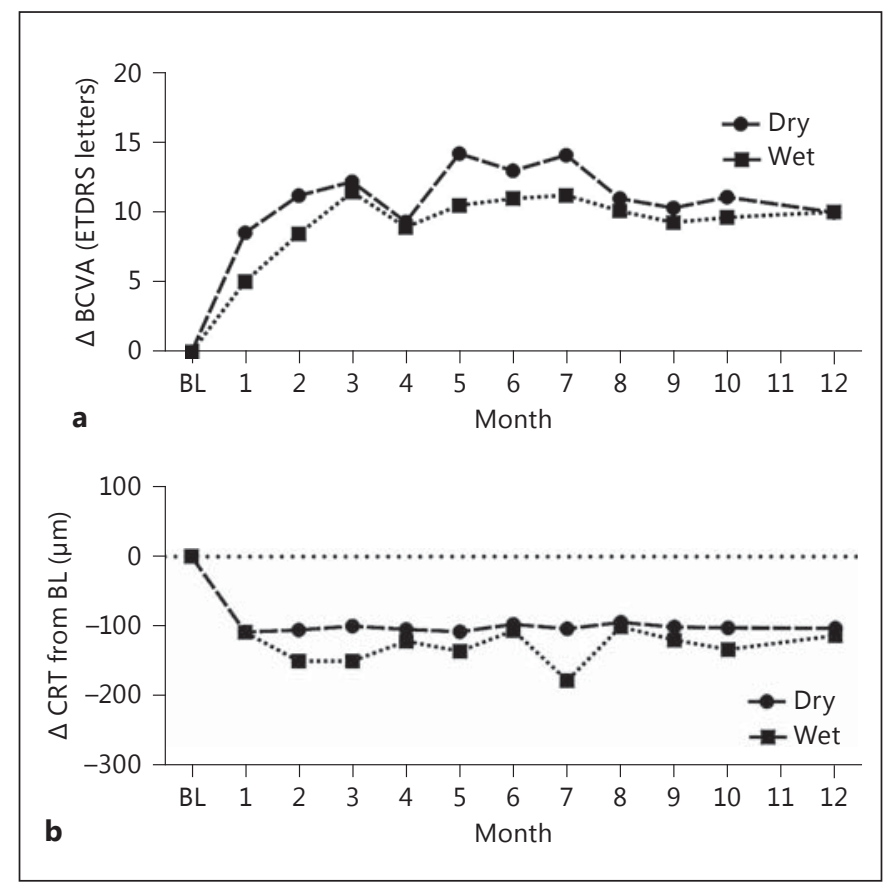

Fig. 3. Mean change in BCVA (a) and CRT (b) from baseline (BL) in patients with recurrent fluid (wet) or without recurrent fluid (dry). Letter gain and decrease in CRT were not statistically different between the subgroups (analysed by one-way ANOVA).

\section{Results}

Twenty-six patients were included in this study. The mean patient age at the time of diagnosis of exudative AMD was 80 years (range 54-93, 14 female patients). The mean BCVA at baseline was 52 ETDRS letters (range $5-80$ ), and the CRT was $413 \mu \mathrm{m}$ (range 242-760). BCVA increased from a mean of $52 \pm 4$ (SEM) letters at baseline to $63 \pm 4$ at 12 months $(\mathrm{p}=0.003)$. CRT decreased from $413 \pm 24 \mu \mathrm{m}$ at baseline to $305 \pm 23 \mu \mathrm{m}$ at 12 months $(p=0.002)$. The change in BCVA and CRT in our patient cohort is summarized in figure 1 .

At baseline, all patients had either IRF (70\%) or SRF (70\%) or a combination of both (100\%) (fig. 2 a). After the loading phase, consisting of three monthly injections, the percentage of patients displaying IRF was $12 \%$, and the percentage of patients having SRF was $4 \%$ (fig. 2b1, b2). No fluid was detected in $84 \%$ of patients after the loading phase. After switching to the bimonthly fixed maintenance regimen, the percentage of patients displaying SRF or IRF increased between the injections following a seesaw pattern (fig. 2b1, b2). This pattern was more pronounced with IRF than with SRF. Nine out of 26 patients
(34\%) had complete regression of IRF or SRF after the loading phase. Subgroup analysis showed that eyes that remained without fluid after the loading phase responded similarly to aflibercept with regard to vision gain and CRT decrease compared to eyes having recurrent IRF or SRF (fig. 3a, b).

We next analysed morphological changes in the subRPE space. Longitudinal measurements of serous and fibrovascular PEDs showed a marked decrease during the loading phase with aflibercept in both groups $(\mathrm{p}=0.04$ and $\mathrm{p}=0.15$, respectively; fig. $4 \mathrm{a}$ ). Whereas the fibrovascular PED remained fairly stable over the course of the year under a bimonthly fixed regimen, the serous PEDs displayed a seesaw pattern with increase in serous fluid in the treatment-free intervals and a decrease in PED height 1 month after injection (fig. 4b1). Eyes with serous PEDs had a significantly higher decrease in CRT from baseline compared to eyes with fibrovascular PEDs. However, there was only a tendency towards more letter gain in eyes with fibrovascular PEDs compared to eyes with serous PEDs over the course of 1 year (fig. $4 \mathrm{~b} 2$ ). Under treatment with aflibercept, choroidal thickness did not decrease significantly over the course of 1 year (fig. 4c).

Nine eyes $(34 \%)$ had areas of atrophy at baseline (fig. 5a). During treatment, one eye developed atrophy at 1 month and two eyes at 2 months after the initiation of treatment with intravitreal aflibercept. At 1 year, 13 eyes (50\%) displayed atrophy within the volume scan area. Subgroup analysis showed that patients without atrophy at 1 year did significantly better in terms of BCVA compared to patients having atrophy $(\mathrm{p}=0.03$; fig. 5b). Thereafter, we analysed whether the presence of either IRF or SRF correlated with the presence of atrophy at the end of year 1 . We found a significant but rather weak correlation of the presence of atrophy with SRF $(r=0.45, p=0.02)$ but not with IRF ( $\mathrm{r}=-0.21$, n.s.).

\section{Discussion}

We report here on functional and anatomical outcomes using a fixed bimonthly treatment regimen for exudative AMD with intravitreal aflibercept. Whereas the pivotal studies of aflibercept used an 8-week (56 days) retreatment interval, the mean treatment interval in our study cohort was 2 months (61 days). The gain in ETDRS letters in our study at 1 year was 9.3 , which is slightly better than the 8.4 seen for the 8 -week aflibercept regimen in the VIEW studies. In our study, there was a low percentage of eyes displaying persisting IRF or SRF after the load- 


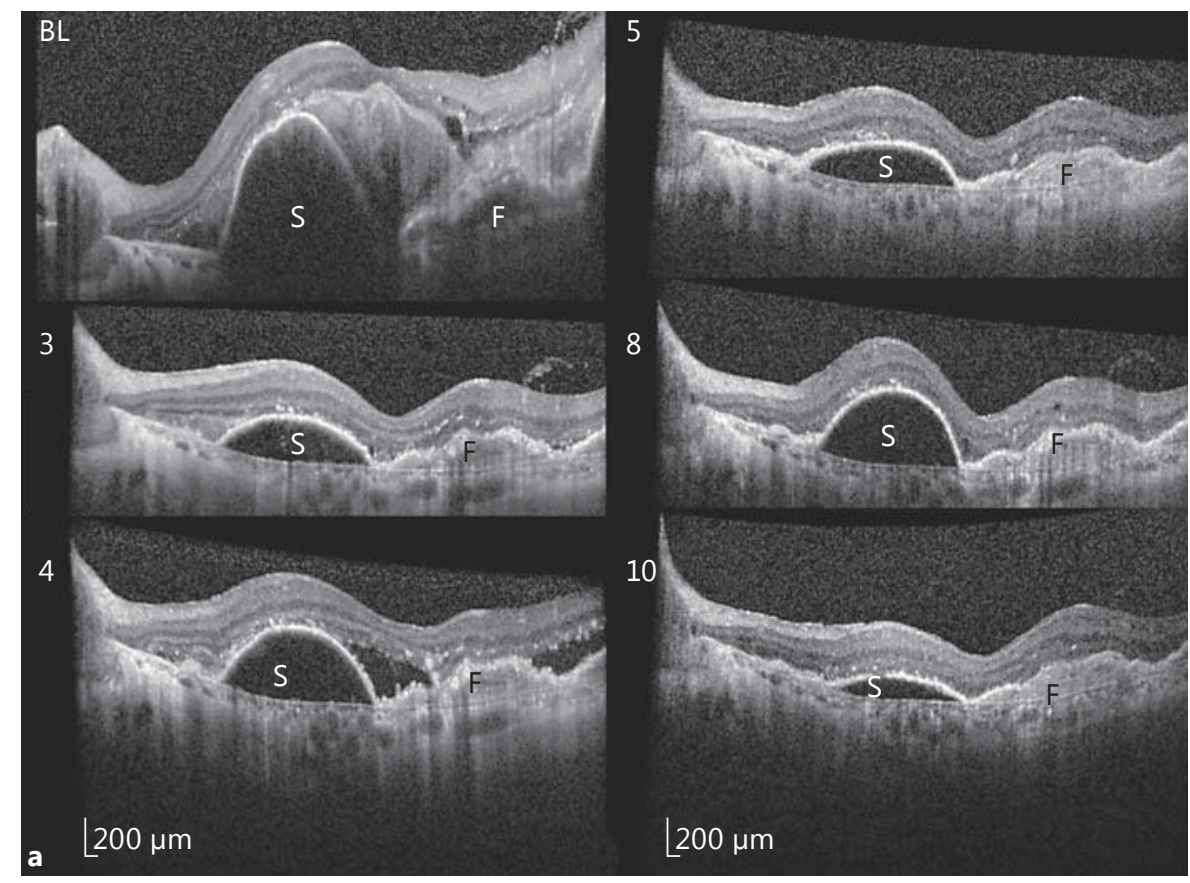

Fig. 4. The response of PED to aflibercept using a fixed treatment regimen is presented. a Representative case history showing combined serous (S) and fibrovascular (F) PED at baseline (BL) and months 3, 4, 5, 8 and 10. b1 Quantitative analysis of PEDs using the horizontal single scan in the rescan registration mode (the baseline was set at 1.0). b2 BCVA gain and CRT decrease at month 12 in patients with serous PED (S; $\mathrm{n}=8)$ and fibrovascular PED $(\mathrm{F} ; \mathrm{n}=12)$ at baseline (unpaired t test; n.s. = not significant). c Quantitative analysis of the mean choroidal thickness at each month.

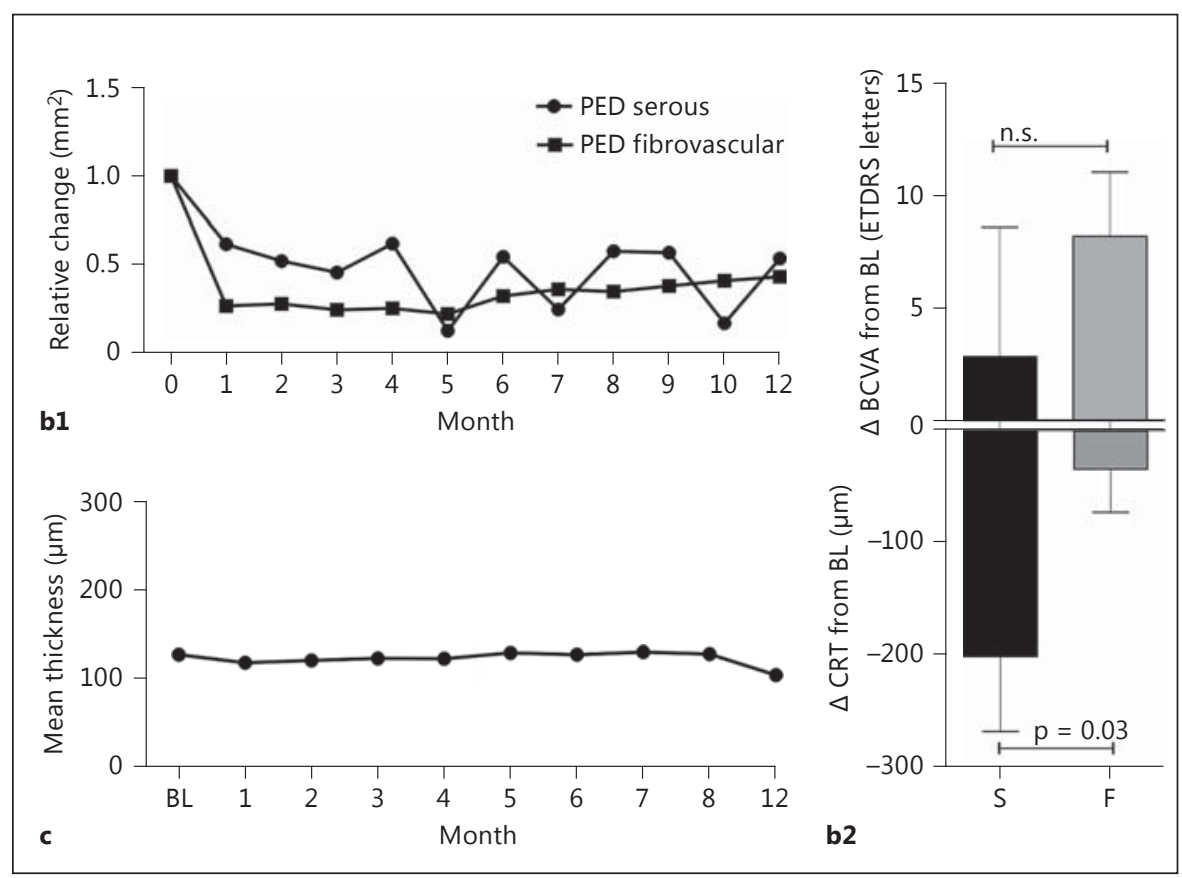

ing phase. After three injections, only $12 \%$ of eyes displayed IRF or SRF on SD-OCT. In the VIEW studies, the proportion of patients with no retinal fluid on time-domain OCT ranged from 60.3 to $72.4 \%$ at week 52, which is in keeping with our results at 1 year [2].

Several salient OCT features merit further discussion, which have not received much attention in the literature so far. In the first instance, our data shows that despite excellent functional visual outcomes, a bimonthly treatment regimen leads not only to fluctuations in IRF and SRF but also in serous PEDs. The response of SRF to intravitreal aflibercept in our patient cohort is very rapid and remains stable during the maintenance phase with extended intervals. However, IRF is fluctuating to a greater extent and seems to be more prone to recurrence during the bimonthly treatment intervals. However, we did 

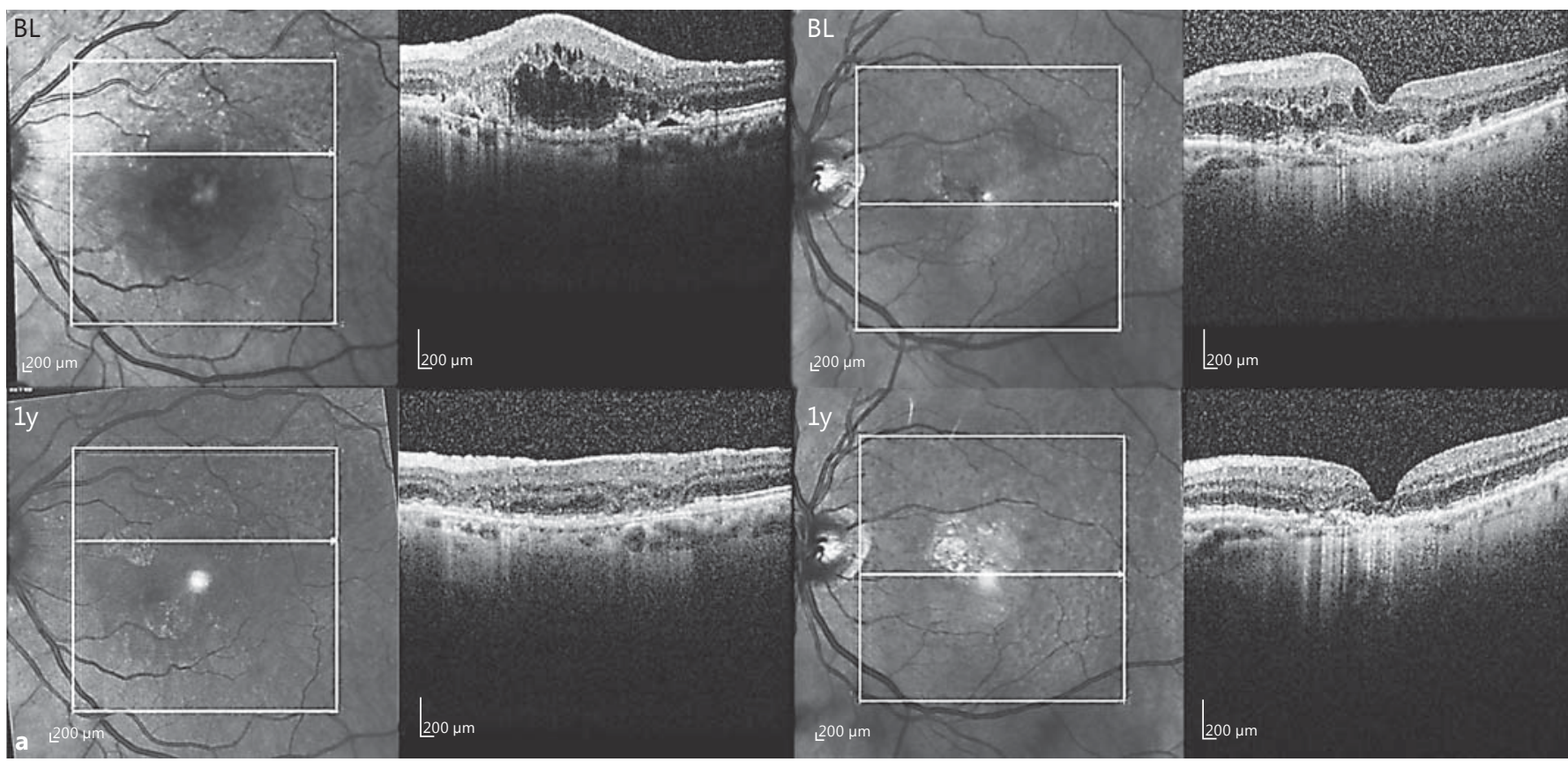

Fig. 5. Presence of atrophy of the RPE and influence on BCVA. a Representative case history showing 2 patients with RPE atrophy at baseline (BL) and at 1 year (1y). b Quantitative analysis of BCVA in patients with and without atrophy at 1 year $(* \mathrm{p}=0.03)$.

not find significant differences in BCVA between eyes with recurrent fluid and eyes without recurrent fluid after the loading phase.

Previous reports on the effect of anti-VEGF treatment of PEDs include ranibizumab and bevacizumab. However, to our knowledge, there is no detailed report on the effect of aflibercept on either serous or fibrovascular PEDs. We showed that serous PEDs respond well to aflibercept and have not found any signs of RPE rips in our patient cohort. However, as with IRF, a bimonthly treatment interval leads to recurrence of serous PEDs between injections. In contrast, fibrovascular PEDs displayed a robust reduction in height during the loading phase and did not show fluctuations thereafter. An interesting finding that needs confirmation in a larger patient cohort is that, although eyes with fibrovascular PEDs

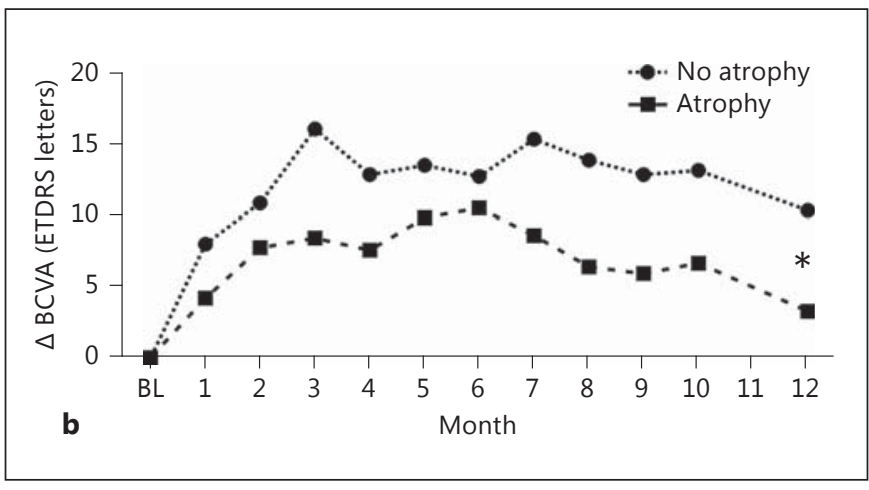

showed less final CRT decrease compared to eyes with serous PEDs or no PEDs at all, the mean visual acuity gain seemed to be slightly better in eyes with fibrovascular PEDs when compared to eyes with serous PEDs.

Another finding of this study is that patients with RPE atrophy had a less favourable course of vision improvement and significantly less BCVA gain at 1 year compared to patients without RPE atrophy. A similar observation has been reported in the 7-year outcome of ranibizumab treatment for exudative AMD [13]. In our study, 3 patients $(11 \%)$ developed atrophy during the 1-year treatment with aflibercept. However, atrophy became visible already after the first injection at month 1 in one eye and after the second injection at month 2 in the other two eyes. It seems to be unlikely that atrophy develops in such a short period of time. Therefore, we assume that atrophy 
was to a large degree pre-existent but masked by IRF or SRF. An intriguing finding is the correlation of SRF with the presence of atrophy at the end of year 1 . This may be due to a more pronounced dysfunction of the RPE, causing accumulation of fluid in the subretinal space as compared to patients with IRF, where structural changes within the retina may play a bigger role.

Our study has several weaknesses, mainly due to its retrospective nature and the relatively small number of subjects. This was in part due to the strict inclusion criteria as all follow-up scans had to be acquired with the rescan registration feature. Furthermore, PED was measured using the horizontal single-line OCT scan in the rescan mode, and therefore measurements only provide an approximate value of the actual PED size.

The VIEW studies have shown that a bimonthly treatment regimen results in excellent functional results. However, on a morphological level, the VIEW studies show that bimonthly injections lead to fluctuations in CRT. These fluctuations in CRT are in keeping with our study. Analysis of central thickness fluctuations in our cohort showed that this was mainly driven by fluid fluctuations in the intraretinal layers as well as the sub-RPE space. On the contrary, bimonthly treatment with aflibercept resulted in excellent BCVA gain over the course of 1 year and, therefore, represents a good treatment option for patients with neovascular AMD.

Clinical data from PRN or treat-and-extend treatment regimens will provide further information on which intervals are optimal for intravitreal aflibercept in order to maintain morphological stability.

In conclusion, using a bimonthly regimen with aflibercept for neovascular AMD, we found marked fluctuations in IRF and SRF as well as serous PEDs.

\section{Disclosure Statement}

A.E. reports honoraria from Bayer for lectures and a travel grant from Allergan. S.W. reports grants from Swiss National Science Foundation (SNSF), non-financial support from Heidelberg Engineering; other from Alcon, Allergan, Bayer Healthcare, Novartis Pharma and Roche, outside the submitted work. M.S.Z. reports grants from Swiss National Science Foundation (SNSF), a grant from Bayer and non-financial support from Heidelberg Engineering; he is a consultant for Allergan, consultant for Bayer Healthcare and stock holder in Novartis Pharma.

\section{References}

1 Congdon NG, Friedman DS, Lietman T: Important causes of visual impairment in the world today. JAMA 2003;290:2057-2060.

2 Schmidt-Erfurth U, Kaiser PK, Korobelnik JF, Brown DM, Chong V, Nguyen QD, Ho AC, Ogura Y, Simader C, Jaffe GJ, Slakter JS, Yancopoulos GD, Stahl N, Vitti R, Berliner AJ, Soo Y, Anderesi M, Sowade O, Zeitz O, Norenberg C, Sandbrink R, Heier JS: Intravitreal aflibercept injection for neovascular age-related macular degeneration: ninety-six-week results of the view studies. Ophthalmology 2014;121:193-201.

3 Heier JS, Brown DM, Chong V, Korobelnik JF, Kaiser PK, Nguyen QD, Kirchhof B, Ho A, Ogura Y, Yancopoulos GD, Stahl N, Vitti R, Berliner AJ, Soo Y, Anderesi M, Groetzbach G, Sommerauer B, Sandbrink R, Simader C, Schmidt-Erfurth U: Intravitreal aflibercept (VEGF trap-eye) in wet age-related macular degeneration. Ophthalmology 2012;119: 2537-2548.

4 Regillo CD, Brown DM, Abraham P, Yue H, Ianchulev T, Schneider S, Shams N: Randomized, double-masked, sham-controlled trial of ranibizumab for neovascular age-related macular degeneration: PIER Study year 1 . Am J Ophthalmol 2008;145:239-248.
5 Schmidt-Erfurth U, Eldem B, Guymer R, Korobelnik JF, Schlingemann RO, Axer-Siegel R, Wiedemann P, Simader C, Gekkieva M, Weichselberger A: Efficacy and safety of monthly versus quarterly ranibizumab treatment in neovascular age-related macular degeneration: the EXCITE study. Ophthalmology 2011;118:831-839.

6 Martin DF, Maguire MG, Ying GS, Grunwald JE, Fine SL, Jaffe GJ: Ranibizumab and bevacizumab for neovascular age-related macular degeneration. N Engl J Med 2011;364:18971908.

7 Martin DF, Maguire MG, Fine SL, Ying GS, Jaffe GJ, Grunwald JE, Toth C, Redford M, Ferris FL 3rd: Ranibizumab and bevacizumab for treatment of neovascular age-related macular degeneration: two-year results. Ophthalmology 2012;119:1388-1398.

8 Economides AN, Carpenter LR, Rudge JS, Wong V, Koehler-Stec EM, Hartnett C, Pyles EA, Xu X, Daly TJ, Young MR, Fandl JP, Lee F, Carver S, McNay J, Bailey K, Ramakanth S, Hutabarat R, Huang TT, Radziejewski C, Yancopoulos GD, Stahl N: Cytokine traps: multi-component, high-affinity blockers of cytokine action. Nat Med 2003;9:47-52.

9 Stewart MW, Rosenfeld PJ: Predicted biological activity of intravitreal VEGF Trap. Br J Ophthalmol 2008;92:667-668.
10 Kumar N, Marsiglia M, Mrejen S, Fung AT, Slakter J, Sorenson J, Freund KB: Visual and anatomical outcomes of intravitreal aflibercept in eyes with persistent subfoveal fluid despite previous treatments with ranibizumab in patients with neovascular age-related macular degeneration. Retina 2013;33:16051612.

11 Stewart MW, Rosenfeld PJ, Penha FM, Wang F, Yehoshua Z, Bueno-Lopez E, Lopez PF: Pharmacokinetic rationale for dosing every 2 weeks versus 4 weeks with intravitreal ranibizumab, bevacizumab, and aflibercept (vascular endothelial growth factor trap-eye). Retina 2012;32:434-457.

12 Lee SY, Stetson PF, Ruiz-Garcia H, Heussen FM, Sadda SR: Automated characterization of pigment epithelial detachment by optical coherence tomography. Invest Ophthalmol Vis Sci 2012;53:164-170.

13 Rofagha S, Bhisitkul RB, Boyer DS, Sadda SR, Zhang K; SEVEN-UP Study Group: Sevenyear outcomes in ranibizumab-treated patients in ANCHOR, MARINA, and HORIZON: a multicenter cohort study (SEVENUP). Ophthalmology 2013;120:2292-2299. 